Pacific Journal of Mathematics

WEAKLY ALMOST PERIODIC SEMIGROUPS OF OPERATORS 


\title{
WEAKLY ALMOST PERIODIC SEMIGROUPS OF OPERATORS
}

\author{
W. M. Ruess AND W. H. Summers
}

\begin{abstract}
We address the question as to when a motion or almost-orbit $u$ of a strongly continuous semigroup $(S(t))_{t \geq 0}$ of operators in a Banach space $X$ will be weakly almost periodic in the sense of Eberlein. In particular, we show (a) that this is the case in practice exactly when $u$ uniquely decomposes as the sum $u=S(\cdot) y+\varphi$ of an almost periodic motion $S(\cdot) y: \mathbb{R}^{+} \rightarrow X$ of $(S(t))_{t \geq 0}$ and a function $\varphi: \mathbb{R}^{+} \rightarrow X$ that vanishes at infinity in a certain weak sense, and (b) that an almost-orbit $u$ of a uniformly bounded $C_{0}$-semigroup of linear operators will be weakly almost periodic provided only that $u$ has weakly relatively compact range. Our results on existence and representation are then applied to a qualitative study of asymptotic behavior of solutions to the abstract Cauchy problem in which the focus is on almost periodicity properties and ergodic theorems.
\end{abstract}

Introduction. The point of departure for our work in this paper is the problem of distinguishing properties related to almost periodicity for solutions to the abstract Cauchy problem associated with the generator of a strongly continuous semigroup $(S(t))_{t \geq 0}$ of operators in a Banach space $X$. In the homogeneous case, solutions can be realized as motions of $(S(t))_{t \geq 0}$, and so the study of their asymptotic behavior reduces to a corresponding study of semigroup motions. Since weak or integral solutions to the inhomogeneous problem are almost-orbits of the associated semigroup in many concrete instances, moreover, we include almost-orbits as well as motions of $(S(t))_{t \geq 0}$ within the scope of our investigation.

In [21] (and the survey article [22]), we have treated the problem of characterizing motions that are asymptotically almost periodic; i.e., those for which their set of translates is relatively compact in the sup-normed space $\left(C_{b}\left(\mathbb{R}^{+}, X\right),\|\cdot\|_{\infty}\right)$ of all bounded continuous functions from $\mathbb{R}^{+}$into $X$. Our present purpose is a study of motions and almost-orbits that are weakly almost periodic in the sense of Eberlein [9], which corresponds to the set of translates being relatively compact with respect to the weak topology of the Banach space $\left(C_{b}\left(\mathbb{R}^{+}, X\right),\|\cdot\|_{\infty}\right)$. Following a preliminary section, we show in $\S 2$ that (a) an almost-orbit $u: \mathbb{R}^{+} \rightarrow X$ of $(S(t))_{t \geq 0}$ is weakly almost pe- 
riodic if and only if there exist a unique element $y$ in the weak $\omega$-limit set of $u$ and a uniquely determined Eberlein-weakly almost periodic function $\varphi: \mathbb{R}^{+} \rightarrow X$ with the zero function in the weak closure of its set of translates such that $u=S(\cdot) y+\varphi$ and $S(\cdot) y: \mathbb{R}^{+} \rightarrow X$ is almost periodic (Theorem 2.4 ), and (b) every almost-orbit with weakly relatively compact range of a uniformly bounded $C_{0}$-semigroup of linear operators is weakly almost periodic (Theorem 2.1). In $\S 3$, we then apply our results to the questions of existence and representation of weakly almost periodic and almost periodic solutions to the abstract Cauchy problem.

1. Preliminaries. Throughout the paper, $X$ will denote a (real or complex) Banach space, which we shall tacitly assume to be complex whenever spectral properties of linear operators enter the picture. The dual of $X$ will be denoted by $X^{*}$, and $B_{X^{*}}$ will denote the dual unit ball. For a subset $D$ of $X$, the closure and weak closure of $D$ will be denoted by $\operatorname{cl} D$ and w- $\operatorname{cl} D$, respectively, while co $D$ will denote the convex hull of $D$. Furthermore, $(S(t))_{t \geq 0}$ will hereafter denote a strongly continuous semigroup of operators on a weakly closed subset $C$ of $X$.

For a function $u: \mathbb{R}^{+} \rightarrow X$, we will refer to $\gamma(u)=\{u(t): t \geq 0\}$ as the orbit of $u$ and to $\omega_{w}(u)=\left\{y \in X: \exists 0 \leq t_{n} \rightarrow \infty\right.$ such that $u\left(t_{n}\right) \rightarrow y$ weakly in $\left.X\right\}$ as the weak $\omega$-limit set of $u$. In case $u=S(\cdot) x$ is a semigroup motion through some $x \in C$, however, we shall follow the standard practice of denoting these sets by $\gamma(x)$ and $\omega_{w}(x)$, respectively.

1.1. Definition [19, p. 351]. A continuous function $u: \mathbb{R}^{+} \rightarrow C$ is said to be an almost-orbit of $(S(t))_{t \geq 0}$ if

$$
\lim _{t \rightarrow \infty} \sup _{h \in \mathbb{R}^{+}}\|u(t+h)-S(h) u(t)\|=0
$$

As mentioned in the Introduction, the broader concept of an almostorbit (which might more aptly be termed an almost motion) of a semigroup includes weak or integral solutions to certain classes of the inhomogeneous Cauchy problem associated with a generator $A: \mathscr{D}(A) \subseteq$ $X \rightarrow X$ of a strongly continuous semigroup $(S(t))_{t \geq 0}$ of operators on $C=$ w-cl $\mathscr{D}(A)$, and we pause to note two specific instances:

1.1.1. Assume that $(S(t))_{t \geq 0}$ is a uniformly bounded $C_{0}$-semigroup of continuous linear operators on $X$, and let $x \in X$. If $f \in L^{1}\left(\mathbb{R}^{+}, X\right)$, 
then it readily follows that the function

$$
u(t)=S(t) x+\int_{0}^{t} S(t-s) f(s) d s
$$

is an almost-orbit of $(S(t))_{t \geq 0}$. In particular, mild solutions of the inhomogeneous Cauchy problem

$$
\left\{\begin{array}{l}
\dot{u}(t)=A u(t)+f(t), \quad t>0, \\
u(0)=x \in X
\end{array}\right.
$$

are almost-orbits of $(S(t))_{t \geq 0}$ whenever $f \in L^{1}\left(\mathbb{R}^{+}, X\right)$.

1.1.2. Assuming that $X$ is uniformly convex and $A$ is $m$-dissipative in $X$, if $f \in L^{1}\left(\mathbb{R}^{+}, X\right)$, then the integral solution (in the sense of Bénilan [3]) to the Cauchy problem

$$
\left\{\begin{array}{l}
\dot{u}(t) \in A u(t)+f(t), \quad t>0, \\
u(0)=x \in C
\end{array}\right.
$$

is an almost-orbit of the contraction semigroup $(S(t))_{t \geq 0}[19$, p. 362].

The spaces of all bounded continuous functions from $\mathbb{R}^{+}$into $X$ and from $\mathbb{R}$ into $X$ will be denoted by $C_{b}\left(\mathbb{R}^{+}, X\right)$ and $C_{b}(\mathbb{R}, X)$, respectively, and we shall hereafter assume that each of these spaces is equipped with the supremum norm. For $J \in\left\{\mathbb{R}^{+}, \mathbb{R}\right\}, f \in C_{b}(J, X)$, and $\omega \in J$, moreover, we put $f_{\omega}(t)=f(t+\omega), t \in J$, and let $H(f)=\left\{f_{\omega}: \omega \in J\right\}$ denote the set of all translates of $f$.

1.2. Definition. (a) A function $f \in C_{b}(\mathbb{R}, X)$ is said to be almost periodic (a.p.) if $H(f)$ is relatively compact in $C_{b}(\mathbb{R}, X)$.

(b) A function $f \in C_{b}\left(\mathbb{R}^{+}, X\right)$ is said to be

(i) asymptotically almost periodic (a.a.p.) if $H(f)$ is relatively compact in $C_{b}\left(\mathbb{R}^{+}, X\right)$, and

(ii) Eberlein-weakly almost periodic (E.-w.a.p.) if $H(f)$ is weakly relatively compact in $C_{b}\left(\mathbb{R}^{+}, X\right)$.

Of these notions, (a) dates back to Bohl, Bohr, and Bochner (cf. [4]), (b) (i) to Fréchet [11, 12] for $\operatorname{dim} X<\infty$ (cf. [31] and [21, 22, 24] for general $X$ ), and (b) (ii) to Eberlein [9] in the scalar case (cf. $[18]$ and $[22,25]$ for general $X)$.

The spaces of all almost periodic, asymptotically almost periodic, and Eberlein-weakly almost periodic functions will be denoted by $A P(\mathbb{R}, X), A A P\left(\mathbb{R}^{+}, X\right)$, and $W\left(\mathbb{R}^{+}, X\right)$, respectively. Further, we shall let $W_{0}\left(\mathbb{R}^{+}, X\right)$ denote the vector subspace of $W\left(\mathbb{R}^{+}, X\right)$ consisting of all $\varphi \in W\left(\mathbb{R}^{+}, X\right)$ for which the zero function belongs to 
the weak closure of $H(\varphi)$, while $C_{0}\left(\mathbb{R}^{+}, X\right)$ will designate the set of those $\varphi \in C_{b}\left(\mathbb{R}^{+}, X\right)$ that vanish at infinity on $\mathbb{R}^{+}$.

For future reference, we shall need the following basic facts concerning the notions of (almost) periodicity defined above.

\subsection{Theorem. Let $u \in C_{b}\left(\mathbb{R}^{+}, X\right)$.}

(a) The following are equivalent:

(i) $u \in A A P\left(\mathbb{R}^{+}, X\right)$;

(ii) there exist uniquely determined functions $g \in A P(\mathbb{R}, X)$ and $\varphi \in C_{0}\left(\mathbb{R}^{+}, X\right)$ such that $u=g \mid \mathbb{R}^{+}+\varphi$.

(b) The following are equivalent:

(i) $u \in W\left(\mathbb{R}^{+}, X\right)$;

(ii) there exist uniquely determined functions $g \in A P(\mathbb{R}, X)$ and $\varphi \in W_{0}\left(\mathbb{R}^{+}, X\right)$ such that $u=g \mid \mathbb{R}^{+}+\varphi$;

(iii) given any sequences $\left(\left(t_{m}, x_{m}^{*}\right)\right)_{m}$ in $\mathbb{R}^{+} \times B_{X^{*}}$ and $\left(\omega_{n}\right)_{n}$ in $\mathbb{R}^{+}$,

$$
\lim _{n} \lim _{m}\left\langle u\left(t_{m}+\omega_{n}\right), x_{m}^{*}\right\rangle=\lim _{m} \lim _{n}\left\langle u\left(t_{m}+\omega_{n}\right), x_{m}^{*}\right\rangle
$$

whenever both iterated limits exist.

Concerning 1.3(a), see Fréchet [11, 12], DeLeeuw and Glicksberg [7], and [22, 24]. The equivalence of (b) (i) and (b) (ii) follows from results in [7], while the equivalence of (b) (i) and (b) (iii) is established in [18] and [25].

In order to place the periodicity properties considered above in perspective-and to motivate the study of these notions in the context of operator semigroups-we make note of their relationship to commonly studied modes of asymptotic behavior for semigroup motions ((a), (d), and (e), below) before closing this preliminary section.

Let $u \in C_{b}\left(\mathbb{R}^{+}, X\right)$, and consider the following assertions:

(a) $\|\cdot\|-\lim _{t \rightarrow \infty} u(t)$ exists;

(b) $u \in A A P\left(\mathbb{R}^{+}, X\right)$;

(c) $u \in W\left(\mathbb{R}^{+}, X\right)$;

(d) $\|\cdot\|-\lim _{T \rightarrow \infty} \frac{1}{T} \int_{0}^{T} u(t) d t$ exists;

(e) $\gamma(u)$ is weakly relatively compact in $X$.

Then (a) implies (b), (b) implies (c), and (c) implies both (d) and (e).

The fact that (c) implies (d) is the extension of the classical ergodic theorem for scalar valued weakly almost periodic functions due to Eberlein [9] to the case of an arbitrary Banach range space given in [27] (also see [26]). The other implications are obvious. 
2. Eberlein-weak almost periodicity for almost-orbits. Our presentation centers around two results. The first (Theorem 2.1) shows that Eberlein-weak almost periodicity arises quite naturally in the context of linear semigroups in that the only requirement for an almost-orbit $u$ of a uniformly bounded $C_{0}$-semigroup of linear operators on $X$ to be E.-w.a.p. is that its orbit $\gamma(u)$ be weakly relatively compact in $X$. (A nonlinear counterpart of this result [26, Thm. 1.4] (also see [23, Théorème 1]) has been established for contraction semigroups in uniformly convex Banach spaces.) The second result (Theorem 2.4), which is valid for general (nonlinear) strongly continuous semigroups, provides a precise representation of E.-w.a.p. almost-orbits as "small" perturbations of (classical) almost periodic motions.

2.1. TheOREM. Assume that $(S(t))_{t \geq 0}$ is a uniformly bounded $C_{0^{-}}$ semigroup of continuous linear operators on $X$, and let $u: \mathbb{R}^{+} \rightarrow X$ be an almost-orbit of $(S(t))_{t \geq 0}$. Then $u$ is Eberlein-weakly almost periodic if and only if $\gamma(u)$ is weakly relatively compact in $X$.

2.2. Corollary. If $(S(t))_{t \geq 0}$ is a uniformly bounded $C_{0}$-semigroup of continuous linear operators on a reflexive Banach space $X$, then every almost-orbit of $(S(t))_{t \geq 0}$ is Eberlein-weakly almost periodic.

Before turning to the proof of Theorem 2.1, some comments are in order.

2.3. Remarks. 1. The assertion of Theorem 2.1 does not carry over to nonlinear operator semigroups, and even fails to hold in the case of contraction semigroups in Hilbert space. To see this, consider the example by Baillon [2] of a contraction semigroup $(S(t))_{t \geq 0}$ in $l_{2}$ generated by $-\partial \varphi$, where $\varphi$ is a convex 1.s.c. function such that $\partial \varphi^{-1}(0) \neq \varnothing$ and, for some $x \in \operatorname{cl} \mathscr{D}(\varphi),\|\cdot\|-\lim _{t \rightarrow \infty} S(t) x$ does not exist. The motion $S(\cdot) x$ in this example is bounded, and thus has weakly relatively compact range, but $S(\cdot) x$ is not E.-w.a.p. Indeed, according to [27], if it were, then $\|\cdot\|-\lim _{T \rightarrow \infty} \frac{1}{T} \int_{0}^{T} S(t) x d t$ would exist. However, Bruck [5, p. 25] has shown that

$$
\lim _{T \rightarrow \infty}\left\|S(T) x-\frac{1}{T} \int_{0}^{T} S(t) x d t\right\|=0
$$

in the setting at hand, which would then yield the desired contradiction.

2. In the context of contraction semigroups on closed convex subsets 
of uniformly convex Banach spaces, however, we have shown [26, Thm. 1.4] (also see [23, Théorème 1]) that a bounded almost-orbit $u$ will be E.-w.a.p. provided that it is asymptotically isometric; i.e., if $\lim _{t \rightarrow \infty}\|u(t+h)-u(t)\|=\rho(h)$ exists uniformly over $h \in \mathbb{R}^{+}$.

3. The literature relating to weak almost periodicity properties of solutions to differential equations (e.g., see [1], [6], [17]) has heretofore dealt only with the notions of either a weakly almost periodic (w.a.p.) function $f \in C_{b}(\mathbb{R}, X)$ (i.e., $x^{*} f \in A P(\mathbb{R})$ for all $x^{*} \in X^{*}$ ) or a weakly asymptotically almost periodic (w.a.a.p.) function $f \in$ $C_{b}\left(\mathbb{R}^{+}, X\right)$ (i.e., $x^{*} f \in A A P\left(\mathbb{R}^{+}\right)$for all $\left.x^{*} \in X^{*}\right)$; we are not aware of any previous results pertaining to Eberlein-weak almost periodicity in the context of differential equations. Contrary to what might have been expected at the outset, however, Theorem 2.1 and [26, Thm. 1.4] cited above taken together with results from [27] and [28] indicate that Eberlein-weak almost periodicity occurs more naturally than weak asymptotic almost periodicity when it comes to motions and almost-orbits of operator semigroups, and can as well lead to stronger conclusions:

(a) In the case of a uniformly bounded linear $C_{0}$-semigroup $(S(t))_{t \geq 0}$ on $X$, a motion through $x \in X$ is E.-w.a.p. provided only that its range $\gamma(x)$ is weakly relatively compact in $X$, whereas it is shown in [28] that $\gamma(x)$ must necessarily be relatively compact with respect to the (generally stronger) topology of uniform convergence on the sets $\left\{S(t)^{*} x^{*}: t \in \mathbb{R}^{+}\right\}, x^{*} \in X^{*}$, in order for $S(\cdot) x$ to be w.a.a.p. For an example where the two notions actually differ, let $X=W\left(\mathbb{R}^{+}\right)$endowed with the sup-norm, and take $(S(t))_{t \geq 0}$ to be the restriction to $X$ of the usual translation semigroup on the space $B U C\left(\mathbb{R}^{+}\right)$of all bounded uniformly continuous functions on $\mathbb{R}^{+}$. Then $\|S(t)\| \leq 1$ for every $t \in \mathbb{R}^{+}$, and every motion $S(\cdot) f: \mathbb{R}^{+} \rightarrow X$ through $f \in$ $W\left(\mathbb{R}^{+}\right)$has weakly relatively compact range (by definition of $W\left(\mathbb{R}^{+}\right)$), whereby every motion is E.-w.a.p. by Theorem 2.1. However, each motion $S(\cdot) g: \mathbb{R}^{+} \rightarrow X$ through some $g \in W\left(\mathbb{R}^{+}\right) \backslash A A P\left(\mathbb{R}^{+}\right)$is obviously not w.a.a.p. Concrete examples of functions belonging to $W\left(\mathbb{R}^{+}\right) \backslash A A P\left(\mathbb{R}^{+}\right)$are given in Examples 3.7 and 3.8 of [25].

(b) According to the ergodic theorem for E.-w.a.p. functions from [27], $\|\cdot\|-\lim _{T \rightarrow \infty} \frac{1}{T} \int_{0}^{T} f(t+h) d t$ exists uniformly over $h \in \mathbb{R}^{+}$whenever $f \in W\left(\mathbb{R}^{+}, X\right)$. Thus, Eberlein-weakly almost periodic functions behave as well as almost periodic functions with respect to convergence of ergodic means. For weakly (asymptotically) almost periodic functions, on the other hand, one can generally only conclude existence 
of the ergodic limit in the weak topology (provided that $X$ is weakly sequentially complete).

Proof of Theorem 2.1. Since an E.-w.a.p. function necessarily has weakly relatively compact range, we need only show that $u$ is E.-w.a.p. when $\gamma(u)$ is weakly relatively compact. To this end, we assume that $\gamma(u)$ is weakly relatively compact in $X$ and choose sequences $\left(\omega_{n}\right)_{n}$ in $\mathbb{R}^{+}$and $\left(\left(t_{m}, x_{m}^{*}\right)\right)_{m}$ in $\mathbb{R}^{+} \times B_{X^{*}}$ so that the limits

$$
\alpha=\lim _{n} \lim _{m}\left\langle u\left(t_{m}+\omega_{n}\right), x_{m}^{*}\right\rangle \text { and } \beta=\lim _{m} \lim _{n}\left\langle u\left(t_{m}+\omega_{n}\right), x_{m}^{*}\right\rangle
$$

both exist. According to Theorem 1.3, it will suffice to show that $\alpha=\beta$.

\section{Case 1. $\left(\omega_{n}\right)_{n}$ is bounded.}

We can thus assume that $\omega_{n} \rightarrow \omega \in \mathbb{R}^{+}$, whereby $\left(u_{\omega_{n}}\right)_{n}$ converges uniformly on $\mathbb{R}^{+}$to $u_{\omega}$ since $u$ is uniformly continuous (cf. [19, p. 351]). From this, we conclude that $\beta=\lim _{m}\left\langle u_{\omega}\left(t_{m}\right), x_{m}^{*}\right\rangle$. Now, given $\varepsilon>0$, choose $n_{0} \in \mathbb{N}$ so that $\left\|u_{\omega_{n}}(t)-u_{\omega}(t)\right\|<\varepsilon$ for all $n \geq n_{0}$ and all $t \in \mathbb{R}^{+}$. Putting $\alpha_{n}=\lim _{m}\left\langle u\left(t_{m}+\omega_{n}\right), x_{m}^{*}\right\rangle, n \in \mathbb{N}$, we have that

$$
\left|\alpha_{n}-\beta\right|=\lim _{m}\left|\left\langle u\left(t_{m}+\omega_{n}\right)-u_{\omega}(t), x_{m}^{*}\right\rangle\right| \leq \varepsilon
$$

for all $n \geq n_{0}$ whence $\alpha=\lim _{n} \alpha_{n}=\beta$.

Case 2. $\left(\omega_{n}\right)_{n}$ is unbounded.

Here, we can assume that $\omega_{n} \rightarrow \infty$, as well as that $\left(u\left(\omega_{n}\right)\right)_{n}$ is weakly convergent to some $x_{0} \in X$ since $\gamma(u)$ is weakly relatively compact. For each $m \in \mathbb{N}$, since $u$ is an almost-orbit of $(S(t))_{t \geq 0}$, we thus have that

$$
\begin{aligned}
\lim _{n} & \left\langle u\left(t_{m}+\omega_{n}\right), x_{m}^{*}\right\rangle \\
& =\lim _{n}\left(\left\langle u\left(t_{m}+\omega_{n}\right)-S\left(t_{m}\right) u\left(\omega_{n}\right), x_{m}^{*}\right\rangle+\left\langle S\left(t_{m}\right) u\left(\omega_{n}\right), x_{m}^{*}\right\rangle\right) \\
& =\left\langle S\left(t_{m}\right) x_{0}, x_{m}^{*}\right\rangle,
\end{aligned}
$$

and hence $\beta=\lim _{m}\left\langle S\left(t_{m}\right) x_{0}, x_{m}^{*}\right\rangle$. Further, since $\left(S\left(t_{m}\right)^{*} x_{m}^{*}\right)_{m}$ is a bounded sequence in $X^{*}$, there exist $x_{0}^{*} \in X^{*}$ and a subnet $\left(S\left(t_{m_{\lambda}}\right)^{*} x_{m_{\lambda}}^{*}\right)_{\lambda}$ of $\left(S\left(t_{m}\right)^{*} x_{m}^{*}\right)_{m}$ which is $w^{*}$-convergent to $x_{0}^{*}$. Now, given $\varepsilon>\hat{0}$, choose $n_{0} \in \mathbb{N}$ so that

(i) $\left|\left\langle u\left(\omega_{n}\right)-x_{0}, x_{0}^{*}\right\rangle\right|<\varepsilon / 2$ when $n \geq n_{0}$ and

(ii) $\left\|u\left(t+\omega_{n}\right)-S(t) u\left(\omega_{n}\right)\right\|<\varepsilon / 2$ for any $n \geq n_{0}$ and all $t \in \mathbb{R}^{+}$. 
Again setting $\alpha_{n}=\lim _{m}\left\langle u\left(t_{m}+\omega_{n}\right), x_{m}^{*}\right\rangle$ for $n \in \mathbb{N}$, if $n \geq n_{0}$, we then have that

$$
\begin{aligned}
\left|\alpha_{n}-\beta\right|= & \lim _{\lambda}\left|\left\langle u\left(t_{m_{\lambda}}+\omega_{n}\right)-S\left(t_{m_{\lambda}}\right) x_{0}, x_{m_{\lambda}}^{*}\right\rangle\right| \\
\leq & \lim _{\lambda}\left|\left\langle u\left(t_{m_{\lambda}}+\omega_{n}\right)-S\left(t_{m_{\lambda}}\right) u\left(\omega_{n}\right), x_{m_{\lambda}}^{*}\right\rangle\right| \\
& \quad+\lim _{\lambda}\left|\left\langle S\left(t_{m_{\dot{\lambda}}}\right) u\left(\omega_{n}\right)-S\left(t_{m_{\lambda}}\right) x_{0}, x_{m_{\lambda}}^{*}\right\rangle\right| \\
\leq & \varepsilon / 2+\left|\left\langle u\left(\omega_{n}\right)-x_{0}, x_{0}^{*}\right\rangle\right|<\varepsilon .
\end{aligned}
$$

Having thus shown that $\alpha=\beta$ in this case as well, the proof of Theorem 2.1 is complete.

In what follows, we shall say that a function $f \in C_{b}\left(\mathbb{R}^{+}, X\right)$ is almost periodic if $f$ is the restriction to $\mathbb{R}^{+}$of an almost periodic function $g \in A P(\mathbb{R}, X)$.

2.4. Theorem. Assume that $(S(t))_{t \geq 0}$ is a strongly continuous semigroup of operators on a weakly closed subset $C$ of $X$, and let $u: \mathbb{R}^{+} \rightarrow$ $C$ be an almost-orbit of $(S(t))_{t \geq 0}$ such that

(*) $S(h) \mid \mathrm{w}-\mathrm{cl} \gamma(u): \mathrm{w}-\mathrm{cl} \gamma(u) \rightarrow X$ is weak-to-weak continuous for each $h \in \mathbb{R}^{+}$.

Then $u \in W\left(\mathbb{R}^{+}, X\right)$ if and only if there exist unique elements $y \in$ $\omega_{w}(u)$ and $\varphi \in W_{0}\left(\mathbb{R}^{+}, X\right)$ such that

(i) $u=S(\cdot) y+\varphi$, and

(ii) $S(\cdot) y$ is almost periodic.

In other words, an almost-orbit $u$ of $(S(t))_{t \geq 0}$ for which $(*)$ holds is E.-w.a.p. if and only if $u$ is a perturbation of an almost periodic motion of $(S(t))_{t \geq 0}$ by a function in $W_{0}\left(\mathbb{R}^{+}, X\right)$. Before proving this, we pause to make some further observations.

2.5. Remarks. 1. Condition (*) in Theorem 2.4 could be replaced by the following formally weaker assumption:

$(* *)$ For any sequence $0 \leq t_{n} \rightarrow \infty$, if $\left(u\left(t_{n}\right)\right)_{n}$ is weakly convergent to some $y \in C$, then $\left(S(h) u\left(t_{n}\right)\right)_{n}$ converges weakly to $S(h) y$ for every $h \in \mathbb{R}^{+}$.

In fact, as the following technical lemma shows, conditions $(*)$ and $(* *)$ are actually equivalent under the circumstances at hand.

Lemma. Assume that $C$ is a weakly closed subset of $X, S: C \rightarrow X$ is continuous, and $u: \mathbb{R}^{+} \rightarrow C$ is a continuous function with weakly 
relatively compact range. Moreover, assume that

(\#) for any sequence $0 \leq t_{n} \rightarrow \infty$, if $\left(u\left(t_{n}\right)\right)_{n}$ converges weakly to some $y \in C$, then $\left(S u\left(t_{n}\right)\right)_{n}$ converges weakly to $S y$.

Then $S \mid \mathrm{w}-\mathrm{cl} \gamma(u): \mathrm{w}-\mathrm{cl} \gamma(u) \rightarrow X$ is weak-to-weak continuous.

Starting from the fact that weakly compact subsets of separable Banach spaces are weakly metrizable (cf. [8, p. 434]), a proof of this lemma follows along elementary lines.

2. Clearly, condition $(*)$ of Theorem 2.4 is always satisfied by a linear $C_{0}$-semigroup $(S(t))_{t \geq 0}$ on $X$ and any almost-orbit $u$ of $(S(t))_{t \geq 0}$. According to [10, Thm. 3.1], and taking the foregoing lemma into account, $(*)$ is likewise satisfied by a (nonlinear) contraction semigroup $(S(t))_{t \geq 0}$ on a reflexive Banach space $X$ and any bounded almost-orbit $u$ provided that $(S(t))_{t \geq 0}$ has an everywhere defined weakly sequentially continuous generator. In view of Remark 2.5.1 and [26, Lemma 1.7], moreover, $(*)$ holds for any contraction semigroup $(S(t))_{t \geq 0}$ on a closed convex subset $C$ of a uniformly convex Banach space $X$ in case $u$ is a bounded almost-orbit that is asymptotically isometric (i.e., such that $\lim _{t \rightarrow \infty}\|u(t+h)-u(t)\|=\rho(h)$ exists uniformly over $\left.h \in \mathbb{R}^{+}\right)$. This latter situation arises in various settings (e.g., see [19, Prop. 5.5 and Cor. 6.2] and [15, §9.3]); we here note only the following one: If $X$ is a Hilbert space, $(S(t))_{t \geq 0}$ is a contraction semigroup on a closed convex subset $C$ of $X, 0 \in C$, and there exists a constant $L \geq 0$ such that

$$
\|S(t) x+S(t) y\|^{2} \leq\|x+y\|^{2}+L\left(\|x\|^{2}-\|S(t) x\|^{2}+\|y\|^{2}-\|S(t) y\|^{2}\right)
$$

for all $t \in \mathbb{R}^{+}$and $x, y \in C$, then every almost-orbit $u$ of $(S(t))_{t \geq 0}$ is asymptotically isometric [19, p. 361]. In particular, the above estimate holds (for $L=0$ ) if $C=-C$ and $(S(t))_{t \geq 0}$ is odd (i.e., $S(t)(-x)=$ $-S(t) x$ for all $t \in \mathbb{R}^{+}$and all $\left.x \in C\right)$.

3. For a uniformly bounded $C_{0}$-semigroup $(S(t))_{t \geq 0}$ of bounded linear operators on $X$, Theorems 2.1 and 2.4 together show that any motion $S(\cdot) x$ with weakly relatively compact range is Eberlein-weakly almost periodic and uniquely decomposes into the sum $S(\cdot) x=S(\cdot) y+$ $\varphi$ of an almost periodic motion $S(\cdot) y$ and a motion $\varphi=S(\cdot)(x-y)$ belonging to $W_{0}\left(\mathbb{R}^{+}, X\right)$. We wish to point out that direct application of the DeLeeuw-Glicksberg theory [7, Thm. 4.11] to the semigroup $(S(t))_{t \geq 0}$ only yields a decomposition of the form $S(\cdot) x=$ $S(\cdot) x_{r}+S(\cdot) x_{0}$ where $S(\cdot) x_{r}$ is almost periodic and $S(\cdot) x_{0}$ is such that $0 \in \mathrm{w}-\mathrm{cl} \gamma\left(x_{0}\right)$. Consequently, even when attention is restricted 
to motions, Theorem 2.1 provides an improvement over direct application of the DeLeeuw-Glicksberg theory through the additional information that the motion $S(\cdot) x_{0}$-and thus $S(\cdot) x$ itself-is actually E.-w.a.p.

Proof of Theorem 2.4. Since the decomposition specified in Theorem 2.4 is certainly sufficient to guarantee that $u \in W\left(\mathbb{R}^{+}, X\right)$, we need only show that it is also necessary, and so let us assume that the almostorbit $u$ is E.-w.a.p. By Theorem 1.3, therefore, there exist uniquely determined functions $g \in A P(\mathbb{R}, X)$ and $\varphi \in W_{0}\left(\mathbb{R}^{+}, X\right)$ such that

(1) $u=g \mid \mathbb{R}^{+}+\varphi$.

As $\varphi \in W_{0}\left(\mathbb{R}^{+}, X\right)$, there exists a sequence $0 \leq \omega_{n} \rightarrow \infty$ such that

(2) $\left(\varphi_{\omega_{n}}\right)_{n}$ converges weakly in $C_{b}\left(\mathbb{R}^{+}, X\right)$ to the zero function. Moreover, we can assume that

(3) $\left(u\left(\omega_{n}\right)\right)_{n}$ is weakly convergent to some $z \in \omega_{w}(u)$, and

(4) $\left(g \omega_{n}\right)_{n}$ converges uniformly on $\mathbb{R}$ to some $f \in A P(\mathbb{R}, X)$.

For each $\varepsilon>0$, since $g \in A P(\mathbb{R}, X)$, there exists a relatively dense subset $P_{\varepsilon}(g)$ of $\mathbb{R}$ such that $\|g(t+\tau)-g(t)\|<\varepsilon$ for each $\tau \in P_{\varepsilon}(g)$ and all $t \in \mathbb{R}[4]$. Thus, choosing $\tau_{n} \in\left[2 \omega_{n}, \infty\right) \cap P_{1 / n}(g), n \in \mathbb{N}$, it is easy to see that

(5) $\left(g_{\tau_{n}}\right)_{n}$ converges to $g$ uniformly on $\mathbb{R}$.

For each $t \in \mathbb{R}^{+}$, taking $(*)$ and the fact that $u$ is an almost-orbit of $(S(t))_{t \geq 0}$ into account, we also have that $\left(S(t) u\left(\omega_{n}\right)\right)_{n}$ converges weakly to $S(t) z$ and $\lim _{n}\left\|u\left(t+\omega_{n}\right)-S(t) u\left(\omega_{n}\right)\right\|=0$. Combining these observations with (1), (2), and (4), we conclude that

(6) $f(t)=S(t) z$ for each $t \in \mathbb{R}^{+}$.

We now claim that

(7) $\left(f_{\tau_{n}}-\omega_{n}\right)_{n}$ converges uniformly on $\mathbb{R}$ to $g$.

Indeed, given $\varepsilon>0$, choose $n_{0} \in \mathbb{N}$ so that $\left\|g_{\omega_{n}}(t)-f(t)\right\|<\varepsilon / 2$ and $\left\|g_{\tau_{n}}(t)-g(t)\right\|<\varepsilon / 2$ for all $t \in \mathbb{R}$ and every $n \geq n_{0}$. Then

$$
\begin{aligned}
& \left\|g(t)-f_{\tau_{n}-\omega_{n}}(t)\right\| \\
& \quad \leq\left\|g(t)-g_{\tau_{n}}(t)\right\|+\left\|g_{\omega_{n}}\left(t+\tau_{n}-\omega_{n}\right)-f\left(t+\tau_{n}-\omega_{n}\right)\right\|<\varepsilon
\end{aligned}
$$

for all $t \in \mathbb{R}$ and every $n \geq n_{0}$.

At this point, setting $y_{n}=S\left(\tau_{n}-\omega_{n}\right) z, n \in \mathbb{N}$, it follows from (6) and (7) that

(8) $\|\cdot\|-\lim _{n} y_{n}=g(0)$.

For each $n \in \mathbb{N}$, moreover, again using $(*)$ and the fact that $u$ is an almost-orbit of $(S(t))_{t \geq 0}$,

$$
y_{n}=\mathrm{w}-\lim _{m} S\left(\tau_{n}-\omega_{n}\right) u\left(\omega_{m}\right)=\mathrm{w}-\lim _{m} u\left(\omega_{m}+\tau_{n}-\omega_{n}\right) \in \omega_{w}(u) .
$$


As we note in the following technical lemma, however, $\omega_{w}(u)$ is weakly closed, and hence

(9) $y=g(0) \in \omega_{w}(u)$.

Finally, according to (6), (7), and (9), we have that

$$
S(t) y=\|\cdot\|-\lim _{n} S(t) y_{n}=\|\cdot\|-\lim _{n} f_{\tau_{n}-\omega_{n}}(t)=g(t)
$$

for each $t \in \mathbb{R}^{+}$, which completes the proof of Theorem 2.4.

2.6. Lemma. If $u: \mathbb{R}^{+} \rightarrow X$ is weakly continuous and $u\left(\mathbb{R}^{+}\right)$is weakly relatively compact in $X$, then $\omega_{w}(u)$ is weakly compact.

Proof. Since there is no loss of generality in replacing $X$ by $\operatorname{cl}\left(\operatorname{span}\left(u\left(\mathbb{R}^{+}\right)\right)\right)$, we may assume that $X$ is separable. Thus, since $K=\mathrm{w}-\mathrm{cl} u\left(\mathbb{R}^{+}\right)$is weakly compact, $K$ is weakly metrizable [8, p. 434]. As $\omega_{w}(u) \subseteq K$, it would therefore suffice to show that $\omega_{w}(u)$ is closed in $(K, d)$, where $d$ is a metric on $K$ that induces the relative weak topology $\sigma\left(X, X^{*}\right) \mid K$, but this is obvious.

REMARK. Lemma 2.6 leads to the following extension of a result by Slemrod [29, Lemma 2.1] for weak (semi)dynamical systems on separable reflexive Banach spaces.

2.7. Proposition. Assume that $\pi: \mathbb{R}^{+} \times X \rightarrow X$ is a weak dynamical system on the Banach space $X$, and that, for some $x \in X, \gamma(x)$ is weakly relatively compact in $X$. Then $\omega_{w}(x)$ is nonvoid, weakly compact, and invariant.

3. Applications and related results. In view of the connection between solutions of abstract Cauchy problems associated with a generator $A$ of a strongly continuous semigroup $(S(t))_{t \geq 0}$ of operators and motions or almost-orbits of $(S(t))_{t \geq 0}$, the assertions of Theorems 2.1 and 2.4 can thus be used to

(1) determine whether such solutions are Eberlein-weakly almost periodic,

and thereby to

(2) obtain existence of (classical) almost periodic solutions even in the absence of a priori norm-compactness assumptions, and

(3) establish the existence of the norm-limit of ergodic means for solutions.

We will begin by illustrating these features, and shall here focus on 
the linear case; the case of abstract Cauchy problems associated with $m$-dissipative (generally multivalued) operators in uniformly convex Banach spaces (see Remark 2.5.2) has been treated in detail in [26].

Throughout the remainder of the section, we assume that $A: \mathscr{D}(A)$ $\subseteq X \rightarrow X$ is the infinitesimal generator of a uniformly bounded $C_{0^{-}}$ semigroup $(S(t))_{t \geq 0}$ of continuous linear operators on $X$. We shall further consider both the homogeneous Cauchy problem

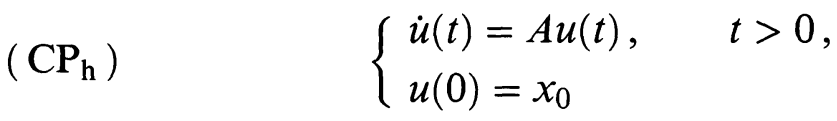

with initial data $x_{0} \in X$ and, for a given function $f \in L^{1}\left(\mathbb{R}^{+}, X\right)$, the inhomogeneous Cauchy problem

$$
\left\{\begin{array}{l}
\dot{u}(t)=A u(t)+f(t), \quad t>0, \\
u(0)=x_{0}
\end{array}\right.
$$

associated with $A$.

It is well known (cf. [13], [20]) that $u=S(\cdot) x_{0}$ is the (unique) strong solution of $\left(\mathrm{CP}_{\mathrm{h}}\right)$ in case $x_{0} \in \mathscr{D}(A)$, and that the mild solution $u(t)=S(t) x_{0}+\int_{0}^{t} S(t-s) f(s) d s$ of $(\mathrm{CP})$ is the candidate for a (classical) solution. As noted in 1.1.1, moreover, every mild solution of (CP) (i.e., the mild solution corresponding to any $x_{0} \in X$ ) is an almost-orbit of $(S(t))_{t \geq 0}$.

3.1. THEOREM. 1. If $\left(\mathrm{CP}_{\mathrm{h}}\right)$ has a strong solution $u$ with weakly relatively compact range, then $u=S(\cdot) x_{0}$ is Eberlein-weakly almost periodic and has a (unique) decomposition $u=S(\cdot) y+\varphi$, where $\varphi \in W_{0}\left(\mathbb{R}^{+}, X\right)$ and $y \in \omega_{w}\left(x_{0}\right)$ is such that $S(\cdot) y$ is almost periodic.

2 . If the mild solution $u$ of $(\mathrm{CP})$ has weakly relatively compact range, then $u$ is Eberlein-weakly almost periodic and has a (unique) decomposition $u=S(\cdot) y+\varphi$, where $\varphi \in W_{0}\left(\mathbb{R}^{+}, X\right)$ and $y \in \omega_{w}(u)$ is such that $S(\cdot) y$ is almost periodic.

3.2. Corollary. 1. Assume that $X$ is reflexive. Then

(a) every strong solution of $\left(\mathrm{CP}_{\mathrm{h}}\right)$ is Eberlein-weakly almost periodic;

(b) if $x_{0} \in \mathscr{D}(A)$, then there exist $y \in \omega_{w}\left(x_{0}\right) \cap \mathscr{D}(A)$ such that the strong solution $u=S(\cdot) y$ of $\left(\mathrm{CP}_{\mathrm{h}}\right)$ for the intial condition $u(0)=y$ is almost periodic;

(c) every mild solution of (CP) is Eberlein-weakly almost periodic.

2. If $(S(t))_{t \geq 0}$ is a differentiable semigroup and $u: \mathbb{R}^{+} \rightarrow X$ is any strong solution of $\left(\mathrm{CP}_{\mathrm{h}}\right)$ with weakly relatively compact range, then 
there exists $y \in \omega_{w}(u)$ such that $S(\cdot) y: \mathbb{R}^{+} \rightarrow X$ is an almost periodic strong solution of $\left(\mathrm{CP}_{\mathrm{h}}\right)$ (with initial vector $x_{0}=y$ ).

As Corollary 3.2 makes plain, Theorem 3.1 can be used to deduce existence of almost periodic strong solutions of $\left(\mathrm{CP}_{\mathrm{h}}\right)$ without a priori norm-compactness assumptions. We mention that every strong solution of $\left(\mathrm{CP}_{\mathrm{h}}\right)$ with norm-relatively compact range is asymptotically almost periodic [21, Corollary 2.5], which also leads to the existence of almost periodic strong solutions to $\left(\mathrm{CP}_{\mathrm{h}}\right)$ in view of the decomposition of an a.a.p motion of $(S(t))_{t \geq 0}$ [21, p. 150] as the sum of an a.p. motion and a function belonging to $C_{0}\left(\mathbb{R}^{+}, X\right)$.

We now turn to the question of convergence of ergodic means. Instead of just considering the limit of the Cesaro means $\frac{1}{T} \int_{0}^{T} u(t) d t$ of a solution $u$ to either $\left(\mathrm{CP}_{\mathrm{h}}\right)$ or $(\mathrm{CP})$, moreover, we shall take into account means with respect to any strongly regular kernel; a function $Q: \mathbb{R}^{+} \times \mathbb{R}^{+} \rightarrow \mathbb{R}$ is said to be strongly regular if

(i) $\left\{Q(s, \cdot): s \in \mathbb{R}^{+}\right\}$is a bounded subset of $L^{1}\left(\mathbb{R}^{+}\right)$,

(ii) $\lim _{s \rightarrow \infty} \int_{0}^{\infty} Q(s, t) d t=1$,

(iii) $\lim _{s \rightarrow \infty} \int_{0}^{T}|Q(s, t)| d t=0$ for each $T>0$, and

(iv) $\lim _{s \rightarrow \infty} \int_{0}^{\infty}|Q(s, t+h)-Q(s, t)| d t=0$ for all $h \in \mathbb{R}^{+}$.

3.3. Theorem. Assume that $(S(t))_{t \geq 0}$ is a uniformly bounded $C_{0^{-}}$ semigroup of continuous linear operators on $X$, and let $u: \mathbb{R}^{+} \rightarrow X$ be an almost-orbit of $(S(t))_{t \geq 0}$ with weakly relatively compact range. Then there exists $z \in \mathrm{cl} \operatorname{co} \omega_{w}(u)$ such that

$$
\|\cdot\|-\lim _{s \rightarrow \infty} \int_{0}^{\infty} Q(s, t) u(t+h) d t=z
$$

uniformly over $h \in \mathbb{R}^{+}$for any strongly regular kernel function $Q: \mathbb{R}^{+} \times$ $\mathbb{R}^{+} \rightarrow \mathbb{R}$. Moreover, $z$ is a fixed point of $(S(t))_{t \geq 0}$, and the only such in $\operatorname{cl} \operatorname{co} \omega_{w}(u)$.

Theorem 3.3 is a consequence of Theorem 2.1 taken in conjunction with the strong ergodic theorem for Eberlein-weakly almost periodic functions from [27]. In the special case that $u$ is a motion $S(\cdot) x$ of $(S(t))_{t \geq 0}$ through some $x \in X$, Theorem 3.3 becomes a version for individual motions of the well known mean ergodic theorem for uniformly bounded $C_{0}$-semigroups on reflexive Banach spaces (cf. [13]).

3.4. Corollary. Assume that $u: \mathbb{R}^{+} \rightarrow X$ is either a strong solution of $\left(\mathrm{CP}_{\mathrm{h}}\right)$ or a mild solution of $(\mathrm{CP})$. If $u$ has weakly relatively 
compact range, then there exists a unique fixed point $z$ of $(S(t))_{t \geq 0}$ in $\operatorname{clco} \omega_{w}(u)$ such that

$$
\|\cdot\|-\lim _{s \rightarrow \infty} \int_{0}^{\infty} Q(s, t) u(t+h) d t=z
$$

uniformly over $h \in \mathbb{R}^{+}$for any strongly regular kernel function $Q: \mathbb{R}^{+} \times$ $\mathbb{R}^{+} \rightarrow \mathbb{R}$.

Before taking up further specific applications, we pause to recall the following fact (cf. [19, p. 350]): For $f \in C_{b}\left(\mathbb{R}^{+}, X\right)$ and $x \in X$, if

(i) $f-f_{\omega} \in C_{0}\left(\mathbb{R}^{+}, X\right)$ (respectively, $f-f_{\omega}$ weakly vanishes at infinity on $\mathbb{R}^{+}$) for each $\omega \geq 0$, and

(ii)

$$
\begin{aligned}
\|\cdot\|-\lim _{T \rightarrow \infty} \frac{1}{T} \int_{0}^{T} f(s+h) d s=x \\
\quad\left(\text { respectively, w- } \lim _{T \rightarrow \infty} \frac{1}{T} \int_{0}^{T} f(s+h) d s=x\right)
\end{aligned}
$$

uniformly over $h \in \mathbb{R}^{+}$, then

$$
\|\cdot\|-\lim _{t \rightarrow \infty} f(t)=x \quad\left(\text { respectively, } \mathrm{w}-\lim _{t \rightarrow \infty} f(t)=x\right) .
$$

Taken together with Theorem 3.3, this leads to the following result.

3.5. Corollary. Assume that $(S(t))_{t \geq 0}$ is a uniformly bounded $C_{0}$-semigroup of continuous linear operators on $X$, and let $u$ : $\mathbb{R}^{+} \rightarrow X$ be an almost-orbit of $(S(t))_{t \geq 0}$ with weakly relatively compact range. If $\lim _{t \rightarrow \infty}\|u(t+h)-u(t)\|=0$ (respectively, $w$ - $\left.\lim _{t \rightarrow \infty}(u(t+h)-u(t))=0\right)$ for each $h \in \mathbb{R}^{+}$, then $\|\cdot\|-\lim _{t \rightarrow \infty} u(t)$ (respectively, $\mathrm{w}-\lim _{t \rightarrow \infty} u(t)$ ) exists and equals $\|\cdot\|-\lim _{T \rightarrow \infty} \frac{1}{T} \int_{0}^{T} u(t) d t$.

In case the uniformly bounded $C_{0}$-semigroup $(S(t))_{t \geq 0}$ generated by $A$ is the restriction to $\mathbb{R}^{+}$of an analytic semigroup of type $(\alpha, M)$ [13, p. 33], $\|t A S(t) x\| \leq M\|x\|$ for each $t>0$ and every $x \in X$, whereby it easily follows that, given any $x \in X$,

$$
\lim _{t \rightarrow \infty}\|S(t+h) x-S(t) x\|=0
$$

for each $h \in \mathbb{R}^{+}$. Since the restriction to $\mathbb{R}^{+}$of any analytic semigroup of type $(\alpha, M)$ is necessarily uniformly bounded, this observation coupled with [19, Prop. 6.3, p. 361] and Corollary 3.5 yields the 
next assertion, which includes a version for individual motions of a known result for analytic semigroups on reflexive Banach spaces (cf. [13, Exercise 17, p. 61]).

3.6. Corollary. Assume that $(S(t))_{t \geq 0}$ is a $C_{0}$-semigroup of bounded linear operators on $X$ which is the restriction to $\mathbb{R}^{+}$of an analytic semigroup of type $(\alpha, M)$, and let $u: \mathbb{R}^{+} \rightarrow X$ be an almostorbit of $(S(t))_{t \geq 0}$. If $u\left(\mathbb{R}^{+}\right)$is weakly relatively compact in $X$, then $\|\cdot\|-\lim _{t \rightarrow \infty} u(t)$ exists and equals $\|\cdot\|-\lim _{T \rightarrow \infty} \frac{1}{T} \int_{0}^{T} u(t) d t$.

Concerning stability properties of the $C_{0}$-semigroup $(S(t))_{t \geq 0}$ generated by $A$, additional information about the point spectrum $\sigma_{p}(A)$ of $A$ leads to the following result.

3.7. Proposition. Assume that $A$ is the infinitesimal generator of a uniformly bounded $C_{0}$-semigroup $(S(t))_{t \geq 0}$ of continuous linear operators on $X$, and let $u: \mathbb{R}^{+} \rightarrow X$ be an almost-orbit of $(S(t))_{t \geq 0}$ with weakly relatively compact range. If $\sigma_{p}(A) \cap i \mathbb{R}=\varnothing$, then $u \in$ $W_{0}\left(\mathbb{R}^{+}, X\right)$. In particular, $\|\cdot\|-\lim _{T \rightarrow \infty} \frac{1}{T} \int_{0}^{T} u(t+h) d t=0$ uniformly over $h \in \mathbb{R}^{+}$whenever $\sigma_{p}(A) \cap i \mathbb{R}=\varnothing$.

Remark. For the connection between Proposition 3.7 and stability of $(S(t))_{t \geq 0}$, recall that $(S(t))_{t \geq 0}$ is said to be strongly (respectively, weakly) stable if $\|\cdot\|-\lim _{t \rightarrow \infty} S(t) x=0$ (respectively, $\left.\mathrm{w}-\lim _{t \rightarrow \infty} S(t) x=0\right)$ for each $x \in X$. This, in turn, is equivalent to requiring that, given any $x \in X$, there exists a sequence $0 \leq \omega_{n} \rightarrow \infty$ such that the corresponding sequence $\left(S\left(\cdot+\omega_{n}\right) x\right)_{n}$ of translates converges to the zero function in $C_{b}\left(\mathbb{R}^{+}, X\right)$ (respectively, uniformly in $C_{b}\left(\mathbb{R}^{+}, X_{w}\right)$, where $X_{w}$ denotes $X$ with its associated weak topology $\left.\sigma\left(X, X^{*}\right)\right)$. According to Proposition 3.7, if $\sigma_{p}(A) \cap i \mathbb{R}=\varnothing$ and $u$ is any almost-orbit of $(S(t))_{t \geq 0}$ with weakly relatively compact range, then there does exist a sequence $0 \leq \omega_{n} \rightarrow \infty$ such that $\left(u_{\omega_{n}}\right)_{n}$ converges to the zero function with respect to the weak topology of $C_{b}\left(\mathbb{R}^{+}, X\right)$. Of course, the condition $\sigma_{p}(A) \cap i \mathbb{R}=\varnothing$ is necessary for the weak stability of $(S(t))_{t \geq 0}$.

Proof of Proposition 3.7. From Theorems 2.1 and 2.4, we know that $u=S(\cdot) y+\varphi$, where $\varphi \in W_{0}\left(\mathbb{R}^{+}, X\right)$ and $y \in \omega_{w}(u)$ is such that $S(\cdot) y$ is almost periodic. For $\mu \in \mathbb{R}$, let

$$
a(y, \mu)=\|\cdot\|-\lim _{T \rightarrow \infty} \frac{1}{T} \int_{0}^{T} e^{-i \mu t} S(t) y d t
$$


be the corresponding Fourier coefficient of (the almost periodic function) $S(\cdot) y$. It is then easy to check that $e^{-i \mu t} S(t) a(y, \mu)=a(y, \mu)$ for all $t \in \mathbb{R}^{+}$, and hence $a(y, \mu)=0$ in view of the assumption on $\sigma_{p}(A)$. Since $a(y, \mu)=0$ for each $\mu \in \mathbb{R}, S(t) y=0$ for every $t \in \mathbb{R}^{+}$, and thus $u \in W_{0}\left(\mathbb{R}^{+}, X\right)$. Since this implies that $0 \in \omega_{w}(u)$, the final assertion now follows as an immediate consequence of Theorem 3.3.

3.8. Corollary. Assume that $X$ is reflexive, and let $A$ be the infinitesimal generator of a uniformly bounded $C_{0}$-semigroup $(S(t))_{t \geq 0}$ of continuous linear operators on $X$. Then the following are equivalent:

(a) $\sigma_{p}(A) \cap i \mathbb{R}=\varnothing$;

(b) every almost-orbit of $(S(t))_{t \geq 0}$ belongs to $W_{0}(\mathbb{R}, X)$;

(c) $S(\cdot) x \in W_{0}\left(\mathbb{R}^{+}, X\right)$ for each $x \in X$;

(d) $\liminf _{t \rightarrow \infty}\left|\left\langle S(t) x, x^{*}\right\rangle\right|=0$ for each $x \in X$ and all $x^{*} \in X^{*}$.

Corollary 3.8 includes $[14, \mathrm{Thm} .2]$ as a special case. In much the same way, our approach also yields a short proof (of an extension) of [14, Thm. 1].

3.9. Corollary. Assume that $A$ is the infinitesimal generator of a uniformly bounded $C_{0}$-semigroup $(S(t))_{t \geq 0}$ of continuous linear operators on $X$ such that $\sigma_{p}(A) \cap i \mathbb{R}=\bar{\varnothing}$ and let $u: \mathbb{R}^{+} \rightarrow X$ be an almost-orbit of $(S(t))_{t \geq 0}$ with weakly relatively compact range. If $x^{*} u \in A A P\left(\mathbb{R}^{+}\right)$for some $x^{*} \in X^{*}$, then $x^{*} u \in C_{0}\left(\mathbb{R}^{+}\right)$.

Proof. According to Theorem 1.3, we have that $x^{*} u=g \mid \mathbb{R}^{+}+\varphi$, where $g \in A P(\mathbb{R})$ and $\varphi \in C_{0}\left(\mathbb{R}^{+}\right)$, while $x^{*} u \in W_{0}\left(\mathbb{R}^{+}\right)$in view of Proposition 3.7. Consequently, there exists a sequence $0 \leq \omega_{n} \rightarrow \infty$ so that $\left(\left(x^{*} u-\varphi\right)_{\omega_{n}}\right)_{n}$ converges pointwise on $\mathbb{R}^{+}$to the zero function. A routine argument based on the fact that $g \in A P(\mathbb{R})$ now shows that $g$ is identically zero, whereby the proof is complete.

Our final set of applications concerns an abstract version of the principle of local energy decay for solutions of the wave equation in exterior domains as considered in [16, Chap. V], [30], and [14]. More specifically, we give a short and straightforward treatment of (an extension of) the material presented in [14, Section 1.2].

To set the context for these results, we assume that $X$ is a reflexive Banach space, and take $F$ to be a Fréchet space (i.e., a complete 
metrizable locally convex space) in which $X$ is continuously embedded. (In applications, for instance, $X=L^{2}(\Omega)$ and $F=L_{\text {loc }}^{2}(\Omega)$.) Furthermore, $A: \mathscr{D}(A) \subset X \rightarrow X$ will be the infinitesimal generator of a uniformly bounded $C_{0}$-semigroup $(S(t))_{t \geq 0}$ of continuous linear operators on $X$.

3.10. Proposition. Assume that $\sigma_{p}(A) \cap i \mathbb{R}=\varnothing$. Then

$$
F-\lim _{t \rightarrow \infty} S(t) x=0
$$

for each $x \in X$ such that $H(S(\cdot) x)=\left\{S(\cdot+\omega): \omega \in \mathbb{R}^{+}\right\}$is relatively compact in $C_{b}\left(\mathbb{R}^{+}, F\right)$ with respect to the topology of uniform convergence.

Proof. Fixing $x \in X$ so that $H(S(\cdot) x)$ is relatively compact in $C_{b}\left(\mathbb{R}^{+}, F\right)$ with respect to the topology of uniform convergence, it then follows that $S(\cdot) x=g \mid \mathbb{R}^{+}+\varphi$, where $g \in A P(\mathbb{R}, F)$ and $\varphi \in C_{0}\left(\mathbb{R}^{+}, F\right)$ [21, p. 147]. By Corollary 3.8, moreover, $S(\cdot) x \in$ $W_{0}\left(\mathbb{R}^{+}, X\right)$. Thus, since $g \mid \mathbb{R}^{+}=S(\cdot) x-\varphi$, we again have that $g \mid \mathbb{R}^{+}$ is identically zero, and the proof is thereby complete.

3.11. Proposition. Assume that $\sigma_{p}(A) \cap i \mathbb{R}=\varnothing$, and let $x \in X$. If $\gamma(x)$ is relatively compact in $F$, then there exists a sequence $0 \leq \omega_{n} \rightarrow$ $\infty$ such that the corresponding sequence $\left(S\left(\cdot+\omega_{n}\right) x\right)_{n}$ of translates of $S(\cdot) x$ converges to zero in $C_{b}\left(\mathbb{R}^{+}, F\right)$ with respect to the topology of uniform convergence on the compact subsets of $\mathbb{R}^{+}$.

Proof. Given $x \in X$, and again applying Corollary 3.8, $\varphi=S(\cdot) x \in$ $W_{0}\left(\mathbb{R}^{+}, X\right)$, and so there exists a sequence $0 \leq \omega_{n} \rightarrow \infty$ such that $\left(\varphi_{\omega_{n}}\right)_{n}$ converges to the zero function with respect to the weak topology of $C_{b}\left(\mathbb{R}^{+}, X\right)$. Assuming that $\gamma(x)$ is relatively compact in $F$, therefore, we may further suppose that $\left(S\left(t+\omega_{n}\right) x\right)_{n}$ converges to zero in $F$ for each $t \in \mathbb{R}^{+}$. Since $S(\cdot) x: \mathbb{R}^{+} \rightarrow F$ is also uniformly continuous, the desired conclusion now follows readily.

REMARK. Propositions 3.10 and 3.11 extend Theorems 3 and 4 of [14].

In closing, we mention that analogues of our results for semigroups can be deduced for groups of operators through the same techniques, and we state one particular result. 
3.12. Theorem. Assume that $(G(t))_{t \in \mathbb{R}}$ is a uniformly bounded $C_{0^{-}}$ group of continuous linear operators on a Banach space $X$, and let $x \in X$. Then $G(\cdot) x \in W(\mathbb{R}, X)$ if and only if $G(\cdot) x$ has weakly relatively compact range in $X$. In this case, $G(\cdot) x=G(\cdot) y+\varphi$, where $\varphi \in W_{0}(\mathbb{R}, X)$ and $y \in \omega_{w}(X)$ with $G(\cdot) y \in A P(\mathbb{R}, X)$.

\section{REFERENCES}

[1] L. Amerio and G. Prouse, Almost-Periodic Functions and Functional Equations, Van Nostrand, New York, 1971.

[2] J. B. Baillon, Un exemple concernant le comportement asymptotique de la solution $d u$ problème $d u / d t+\partial \varphi(u) \ni 0$, J. Funct. Anal., 28 (1978), 369-376.

[3] P. Bénilan, Solutions intégrales d'équations d'évolution dans un espace de Banach, C. R. Acad. Sci. Paris, Sér A, 274 (1972), 47-50.

[4] S. Bochner, Abstrakte fastperiodische Funktionen, Acta Math., 61 (1933), 149184.

[5] R. E. Bruck, Asymptotic convergence of nonlinear contraction semigroups in Hilbert space, J. Funct. Anal., 18 (1975), 15-26.

[6] _ On the weak asymptotic almost-periodicity of bounded solutions of $u^{\prime \prime} \in$ $A u+f$ for monotone A, J. Differential Equations, 37 (1980), 309-317.

[7] K. DeLeeuw and I. Glicksberg, Applications of almost periodic compactifications, Acta Math., 105 (1961), 63-97.

[8] N. Dunford and J. T. Schwartz, Linear operators, Part I, Pure and Appl. Math., 7, Interscience, New York, 1958.

[9] W. F. Eberlein, Abstract ergodic theorems and weak almost periodic functions, Trans. Amer. Math. Soc., 67 (1949), 217-240.

[10] W. E. Fitzgibbon, Weakly continuous nonlinear accretive operators in reflexive Banach spaces, Proc. Amer. Math. Soc., 41 (1973), 229-236.

[11] M. Fréchet, Les fonctions asymptotiquement presque-périodiques continues, C. R. Acad. Sci. Paris, 213 (1941), 520-522.

[12] L__ Les fonctions asymptotiquement presque-périodiques, Rev. Sci., 79 (1941), 341-354.

[13] J. A. Goldstein, Semigroups of linear operators and applications, Oxford Math. Monographs, Oxford Univ. Press, New York, 1985.

[14] N. Iwasaki, Local decay of solutions for symmetric hyperbolic systems with dissipative and coercive boundary conditions in exterior domains, Publ. Res. Inst. Math. Sci. Kyoto Univ., 5 (1969), 193-218.

[15] U. Krengel, Ergodic theorems, de Gruyter Studies Math., 6, de Gruyter, Berlin, 1985.

[16] P. D. Lax and R. S. Phillips, Scattering theory, Pure and Appl. Math., 26, Academic Press, New York, 1967.

[17] B. M. Levitan and V. V. Zhikov, Almost periodic functions and differential equations, Cambridge Univ. Press, Cambridge, 1982.

[18] P. Milnes, On vector-valued weakly almost periodic functions, J. London Math. Soc., (2) 22 (1980), 467-472.

[19] I. Miyadera and K. Kobayasi, On the asymptotic behavior of almost-orbits of nonlinear contraction semigroups in Banach spaces, Nonlinear Anal., 6 (1982), 349-365. 
[20] A. Pazy, Semigroups of linear operators and applications to partial differential equations, Appl. Math. Sci., 44, Springer-Verlag, New York, 1983.

[21] W. M. Ruess and W. H. Summers, Minimal sets of almost periodic motions, Math. Ann., 276 (1986), 145-158.

[22] _ Asymptotic almost periodicity and motions of semigroups of operators, Linear Algebra Appl., 84 (1986), 335-351.

[23] __, Presque-périodicité faible et théorème ergodique pour les semi-groupes de contractions non linéaires, C. R. Acad. Sci. Paris, 305 (1987), 741-744.

[24] __ Compactness in spaces of vector valued continuous functions and asymptotic almost periodicity, Math. Nachr., 135 (1988), 7-33.

[25] __ Integration of asymptotically almost periodic functions and weak asymptotic almost periodicity, Dissertationes Math., 279 (1989).

[26] __ Weak almost periodicity and the strong ergodic limit theorem for contraction semigroups, Israel J. Math., 64 (1988), 139-157.

[27] _ Ergodic theorems for semigroups of operators, to appear.

[28] _ Weak asymptotic almost periodicity for semigroups of operators, to appear.

[29] M. Slemrod, Asymptotic behavior of a class of abstract dynamical systems, J. Differential Equations, 7 (1970), 584-600.

[30] C. H. Wilcox, Scattering theory for the d'Alembert equation in exterior domains, Lecture Notes Math. 442, Springer-Verlag, Berlin, 1975.

[31] S. Zaidman, Almost-periodic functions in abstract spaces, Res. Notes Math. 126, Pitman, Boston, 1985.

Received April 22, 1988. The authors gratefully acknowledge partial support by the Deutsche Forschungsgemeinschaft (DFG).

UNIVERSITÄT ESSEN

4300 ESSEN 1

Federal Republic of Germany

AND

UNIVERSITY OF ARKANSAS

FAYETTEVILLE, AR 72701 



\section{PACIFIC JOURNAL OF MATHEMATICS EDITORS}

\author{
V. S. VARADARAJAN \\ (Managing Editor) \\ University of California \\ Los Angeles, CA 90024-1555-05 \\ Herbert Clemens \\ University of Utah \\ Salt Lake City, UT 84112 \\ Thomas ENRIGHT \\ University of California, San Diego \\ La Jolla, CA 92093
}

R. FINN

Stanford University

Stanford, CA 94305

Hermann FlaschKa

University of Arizona

Tucson, AZ 85721

VAughan F. R. Jones

University of California

Berkeley, CA 94720

Steven Kerckhoff

Stanford University

Stanford, CA 94305
RobION KIRBY

University of California

Berkeley, CA 94720

C. C. MOore

University of California

Berkeley, CA 94720

HAROLd STARK

University of California, San Diego La Jolla, CA 92093

\begin{tabular}{|c|c|c|c|c|}
\hline & ASSOCIA & $E \mathrm{EDI}$ & & \\
\hline R. ARENS & $\begin{array}{l}\text { E. F. BECKENBACH } \\
(1906-1982)\end{array}$ & NEUMANN & $\begin{array}{c}\text { F. WoLF } \\
(1904-1989)\end{array}$ & K. Yoshida \\
\hline & SUPPORTIN & INST & IONS & \\
\hline UNIVERSITY & ARIZONA & UNIVERS & OF OREGON & \\
\hline UNIVERSITY & BRITISH COLUMBIA & UNIVE & OF SOUTHEI & CAL \\
\hline CALIFORNIA & STITUTE OF TECHNOLOGY & STANFOI & UNIVERSITY & \\
\hline UNIVERSITY & CALIFORNIA & UNIVERS & OF HAWAII & \\
\hline MONTANA S & TE UNIVERSITY & UNIVER & OF TOKYO & \\
\hline UNIVERSITY & NEVADA, RENO & UNIVER & OF UTAH & \\
\hline NEW MEXIC & TATE UNIVERSITY & WA & N STATE UN & ERSITY \\
\hline OREGON ST & UNIVERSITY & & I OF WASHINC & ON \\
\hline
\end{tabular}




\section{Pacific Journal of Mathematics}

\section{Vol. 143, No. $1 \quad$ March, 1990}

Walter Bergweiler, On the fix-points of composite functions $\ldots \ldots \ldots \ldots \ldots 1$ Aldo Biancofiore, Maria Lucia Fania and Antonio Lanteri, Polarized surfaces with hyperelliptic sections $\ldots \ldots \ldots \ldots \ldots \ldots \ldots \ldots \ldots$

Ciprian Borcea, Deforming varieties of $k$-planes of projective complete

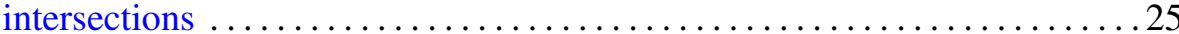

Morton Brown, Fixed points for orientation preserving homeomorphisms of

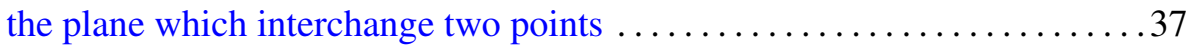

Hao Zhi Chuan, Note on the inequality of the arithmetic and geometric means

Paul Henry Edelman and Dennis E. White, Codes, transforms and the

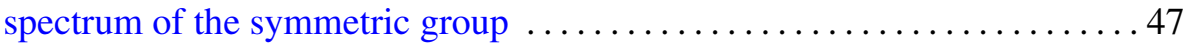

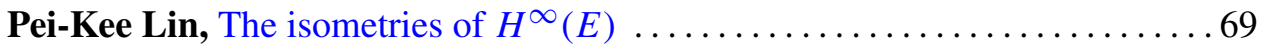

James J. Moloney, Residue class domains of the ring of convergent

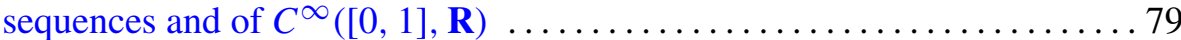

Zhong-Jin Ruan, The structure of pure completely bounded and completely

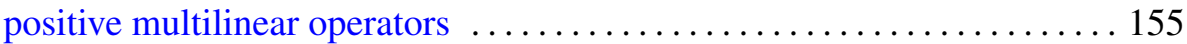

Wolfgang Ruess and William H. Summers, Weakly almost periodic semigroups of operators

Gideon Schwarz, A pretender to the title "canonical Moebius strip" . . . . . . 195

Ryszard Szwarc, Banach algebras associated with spherical representations of the free group 